\title{
Calibration of a Zooming Camera using the Normalized Image of the Absolute Conic
}

\author{
Jean-Yves Guillemaut, Alberto S. Aguado* and John Illingworth \\ School of Electronics and Physical Sciences \\ University of Surrey, Guildford, Surrey, GU2 7XH, UK \\ jean-yves.guillemaut@surrey.ac.uk
}

\begin{abstract}
We present a novel technique for calibrating a zooming camera based on the invariance properties of the Normalized Image of the Absolute Conic (NIAC). First, we show that the camera parameters (independent of the position, orientation and zooming) are determined uniquely by the NIAC. Then, we exploit the invariance properties to develop a stratified calibration method that decouples the calibration parameters. The method is organized in three steps: $i)$ computation of the NIAC, ii) computation of the focal length for each image, iii) computation of the orientation and the position of the camera. The method only requires two images of non-coplanar squares at different orientations and the solution is obtained based on linear minimization that provides fast and stable convergence properties. A finer solution can be obtained by taking the solution of the linear minimisation as a starting point of a non-linear minimization. Preliminary results for synthetic and real data show that the method is capable of obtaining accurate results.
\end{abstract}

\section{Introduction}

The problem of camera calibration consists in determining the geometric properties of the image formation process. In this paper, we consider the problem of determining the geometrical parameters under the assumption of a camera with general motion and zooming. Zooming increases significantly the complexity of the calibration problem making the straightforward use of standard calibration techniques impractical [1]. Several techniques have been proposed aimed at making the calibration of cameras with zoom lenses practical. For example, in [19] the camera parameters with several apertures are estimated by fitting a polynomial at points obtained from fixed zoom settings. Similar methods have been applied for motorised cameras [2] and have been extended for selected zoom apertures [1].

\footnotetext{
* Now at EA UK.
}

Other methods have estimated parameters by using more elaborated approaches. In [13] the calibration is performed by iteratively looking for the best parameters that satisfy the infinite homography constrain [17]. Similar approaches, but exploiting the geometrical properties of non-translating cameras have been presented in $[9,6]$. In these works, nontranslation properties are used to replace descent-based iteration by a linear method. This produces an elegant algorithm for self-calibration under general motions. Unfortunately, these and other self-calibration techniques rely on sufficient and accurate point correspondences. Additionally, self-calibration requires good initial values. Convergence problems and noise have made difficult to translate these techniques into practical applications [16].

The method presented in this paper, capitalises on the invariance properties of the Absolute Conic to simplify calibration equations. Previous works have exploited invariants in calibration. For example, methods have used the image of Points at Infinity which is invariant to translation $[4,5,11]$ or the Image of the Absolute Conic (IAC) which is invariant to changes in position and orientation $[21,11,15,12]$. Our method extends the geometry used on the methods based on the IAC to include invariance to zooming by considering the invariance properties of the Normalized Image of the Absolute Conic (NIAC). First, we show that the camera parameters (independent of the position, orientation and zooming) are determined uniquely by the NIAC. Then, we exploit the invariance properties to develop a stratified method that only requires two views of non-coplanar squares in arbitrary positions. A method that uses planar calibration objects and that includes zooming was presented in [15]. However, this method assumes zero skew, it is not linear and requires an accurate initial estimate of the parameters.

We use the invariance properties of the NIAC to decouple the calibration into three sub-problems: i) intrinsic parameters independent to the zoom (computed through the NIAC), ii) focal length representing the zoom for each image (computed through the IAC for each image), iii) extrinsic parameters for each image. In general, zooming imposes 
a large scale non-linear minimization which is usually unstable and less likely to converge to the solution. The subproblems in our method have small dimensions and the solution can be found linearly. Each sub-problem have dimension four, one and six respectively. If necessary, a final nonlinear minimisation can be done to refine the solution. In this paper, we assume that the position of the principal point does not change with zooming. This has been assumed in other works [15], however according to some studies [20] this is not the case for current cameras technologies. Our preliminary results on real images with relative small zoom factors are encouraging, current work focuses on generalising the method to a variable principal point.

\section{Zooming Camera and the NIAC}

\subsection{The Zooming Camera Model}

We adopt the general pinhole model. In this model, 3D points in the scene $\boldsymbol{P}_{i}=[X, Y, Z, 1]^{\top}$ are mapped into 2D points on the image plane $\boldsymbol{p}_{i}=[u, v, 1]^{\top}$ by a projection

$$
\boldsymbol{p}_{i} \sim M \boldsymbol{P}_{i},
$$

The projection can be represented by a camera model of the form $M=K_{f}\left[\begin{array}{ll}R & t\end{array}\right]$. The extrinsic parameters represent the camera orientation (rotation matrix $R$ ) and position (translation vector $-R^{\top} t$ ) with respect to the world frame. The intrinsic parameters include the coordinates of the principal point $\left(u_{0}, v_{0}\right)$, the scale factors $\alpha_{u}$ and $\alpha_{v}$, and the skew parameter $s$. Physically, the three latter parameters can be expressed in terms of the focal length $f$ in pixel units, the aspect ratio $r$, and the angle $\theta$ defined by the two axes of the camera. Thus,

$$
K_{f}=\left[\begin{array}{ccc}
\alpha_{u} & s & u_{0} \\
& \alpha_{v} & v_{0} \\
& & 1
\end{array}\right]=\left[\begin{array}{ccc}
f & -f \cot \theta & u_{0} \\
& f r \sin \theta & v_{0} \\
& & 1
\end{array}\right] .
$$

The skew parameter $s$, which corresponds to the cosine of the angle $\theta$, is necessary only in the case of CCD cameras ( $s=0$ for digital cameras). In practice, the aspect ratio $r$ is expected to be close to 1 , however this parameter should be computed for an accurate calibration. In the case of a zooming camera, it is convenient to separate the focal length from the other parameters. That is, $K_{f}=K_{1} F_{f}$ with

$$
K_{1}=\left[\begin{array}{ccc}
1 & -\cot \theta & u_{0} \\
& r \sin \theta & v_{0} \\
& & 1
\end{array}\right] \quad \text { and } \quad F_{f}=\left[\begin{array}{lll}
f & & \\
& f & \\
& & 1
\end{array}\right] \text {. }
$$

As such, the general model adopted for a zooming camera is

$$
M=K_{1} F_{f}\left[\begin{array}{ll}
R & \boldsymbol{t}
\end{array}\right] .
$$

We have assumed that zooming is equivalent to varying the focal length of the camera and that the other parameters are
Table 1. A hierarchy of invariants and their properties. The plane at infinity is invariant to translation. The IAC extends the invariance properties to rotation. Ultimately, the NIAC includes zooming as well.

\begin{tabular}{cc}
\hline \hline invariant & motion \\
\hline image of Plane at Infinity & translation \\
\hline IAC & translation, rotation \\
\hline NIAC & translation, rotation, zoom \\
\hline \hline
\end{tabular}

independent of zooming. More elaborated models consider a varying principal point or define a centre of zooming [20]. The model adopted here is usually a reasonable approximation for good quality cameras and relatively small zoom factors.

\subsection{The Inverse Image Formation and the Use of Invariants}

Typically, the camera parameters should be recovered from a set of $3 \mathrm{D}$ control points $\boldsymbol{P}_{i}$ with known positions and its projection in images $\boldsymbol{p}_{i}$. The inverse image formation problem consists in finding the set of values that minimises the cost function

$$
\sum_{i} d\left(\boldsymbol{p}_{i}, K_{1} F_{f}\left[\begin{array}{ll}
R & \boldsymbol{t}
\end{array}\right] \boldsymbol{P}_{i}\right)^{2}
$$

where $d$ defines a geometric distance or error. This can be extended to a sequence of images. By denoting as $j$ the image frame, the cost function becomes

$$
\sum_{j} \sum_{i} d\left(\boldsymbol{p}_{i}^{j}, K_{1} F_{f_{i}}\left[\begin{array}{ll}
R^{j} & \boldsymbol{t}^{j}
\end{array}\right] \boldsymbol{P}_{i}\right)^{2} .
$$

It is tempting to increase the number of views in order to provide more correspondences and increase the robustness of the method. However, by increasing the number of views, we also increase the number of unknowns. In total, a set of $n$ images with different zoom leads to $4+(1+6) n$ unknowns ( 4 intrinsic constant parameters in $K_{1}, 1$ focal length (zoom) in $F_{f_{i}}$ and 6 extrinsic parameters in $R^{j}$ and $\boldsymbol{t}^{j}$ for each image).

The notion of invariant is fundamental if one is interested in increasing the calibration data without increasing the dimensionality of the problem. For example, parallel lines intersect in Points at Infinity whose projections, called vanishing points, are independent to the translation of the camera $[4,5,11]$. Translation can then be used to increase the calibration data without increasing the dimensionality of the problem [8]. This requires to be able to generate an accurate translation motion. The IAC $[21,11,15,12]$ offers more flexibility as it is invariant to position and orientation. 
For zooming a new invariant can be included. The NIAC is invariant to the camera position, orientation and zoom. It occupies the last position in the hierarchy of the invariants by offering more general invariants than the image of the plane at infinity or the image of the absolute conic (see Table 1).

\subsection{The NIAC}

The Absolute Conic $\Omega_{\infty}$ is represented by the $3 \times 3$ identity matrix, i. e. it is defined by the equations $X^{2}+Y^{2}+$ $Z^{2}=0$ and $Z=0$. The Absolute Conic is an imaginary object (it is equivalent to a circle of radius -1 ) located in the plane at infinity $\pi_{\infty}$ (with equation $Z=0$ ). The IAC is also an imaginary conic which is represented by $\omega_{f}=\left(K_{f} K_{f}^{\top}\right)^{-1}$. Before defining the NIAC's zooming invariance, we consider two properties of the set of Images of the Absolute Conic (IACs) for a zooming camera. The first result concerns the concentricity of the IACs, the second property concern the fact that all the IACs are homothetically equivalent.

Result 1 (Concentricity of the IACs) The IACs remain concentric while the camera is zooming. They are centered at the principal point $\left(u_{0}, v_{0}\right)$ of the camera.

Proof The centre of a conic is defined as its pole to the line at infinity $\boldsymbol{l}_{\infty}=(0,0,1)^{\top}$. The result is straightforward by direct computation of the centre $\boldsymbol{C}_{f}$ of the IAC $\omega_{f} . \boldsymbol{C}_{f}$ and $\boldsymbol{l}_{\infty}$ are pole-polar with respect to $\omega_{f}$, so we have $\boldsymbol{l}_{\infty} \sim \omega_{f} \boldsymbol{C}_{f}$. Equivalently in terms of the dual image of the absolute conic $\omega_{f}^{*}=\omega_{f}^{-1}=K_{f} K_{f}^{\top}$, we have $\boldsymbol{C}_{f} \sim \omega_{f}^{*} \boldsymbol{l}_{\infty} \sim K_{f} K_{f}^{\top} \boldsymbol{l}_{\infty}$. The right hand-side term simplifies into

$$
\begin{gathered}
{\left[\begin{array}{ccc}
f & -f \cot \theta & u_{0} \\
& f r \sin \theta & v_{0} \\
& 1
\end{array}\right]\left[\begin{array}{ccc}
f & \\
-f \cot \theta & f r \sin \theta \\
u_{0} & v_{0} & 1
\end{array}\right]\left(\begin{array}{l}
0 \\
0 \\
1
\end{array}\right)} \\
=\left[\begin{array}{ccc}
f & -f \cot \theta & u_{0} \\
& f r \sin \theta & v_{0} \\
& 1
\end{array}\right]\left(\begin{array}{l}
0 \\
0 \\
1
\end{array}\right)=\left(\begin{array}{l}
u_{0} \\
v_{0} \\
1
\end{array}\right) .
\end{gathered}
$$

Finally we have $\boldsymbol{C}_{f} \sim\left(u_{0}, v_{0}, 1\right)^{\top}$, which does not depend on any extrinsic parameter nor the focal length. This completes the proof.

Result 2 (Homotheticity of the IACs) The IACs are all homothetically equivalent when the focal length of the camera varies.

Proof By definition, a homothety is a similarity transformation that preserves orientation, i.e. the composition of a translation, a rotation and an isotropic scaling. Let $\omega_{f}$ be the IAC for the focal length $f$. We want to show that there exists an homothety $H_{f}$, transforming the conic $\omega_{1}$ into $\omega_{f}$,

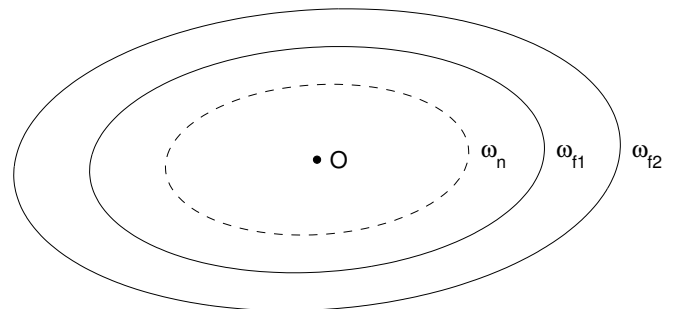

Figure 1. Illustration of the transformation of the IAC while the camera is zooming. The different IACs $\omega_{f_{i}}$ are all centered in the principal point $O$ and are homothetically equivalent to the NIAC $\omega_{n}$. For illustration purpose, the Absolute Conic has been replaced by the diagonal matrix with diagonal values $(1,1,-1)$ so that the images by a camera are real ellipses rather than imaginary entities.

i.e. such that $\omega_{f} \sim H_{f}^{-\top} \omega_{1} H_{f}^{-1}$. Noting that $K_{f}$ can be decomposed in the form

$$
K_{f}=H_{f} K_{1} \quad \text { with } \quad H_{f}=\left[\begin{array}{ll}
f & (1-f) u_{0} \\
& f \quad(1-f) v_{0} \\
& 1
\end{array}\right]
$$

we derive that

$$
\begin{aligned}
\omega_{f} & =\left(K_{f} K_{f}^{\top}\right)^{-1}=\left(H_{f} K_{1} K_{1}^{\top} H_{f}^{\top}\right)^{-1} \\
& =H_{f}^{-\top}\left(K_{1} K_{1}^{\top}\right)^{-1} H_{f}^{-1}=H_{f}^{-\top} \omega_{1} H_{f}^{-1}
\end{aligned}
$$

This completes the proof.

Definition 1 (Normalised Image of the Absolute Conic) The Normalized Image of the Absolute Conic (NIAC) is the Image of the Absolute Conic (IAC) corresponding to a focal length of 1 . It is represented by the symmetric matrix $\omega_{n}=\left(K_{1} K_{1}^{\top}\right)^{-1}$. It is by construction invariant to the position, orientation and zooming of the camera.

In the next section, we show how this invariant can be used for the calibration of a zooming camera.

\section{Application to Camera Calibration}

The invariance of the NIAC to translation, rotation and zoom is the core of the new camera calibration algorithm. The algorithm can be broken into three stages (see Algorithm 1). In the first stage, the NIAC is used to compute the invariant parameters, i.e. the principal point, the aspect ratio and the skew parameter. In the second stage, the focal length, which represents the zooming effect, is recovered separately for each image. In the third stage, the extrinsic parameters, i.e. position and orientation are recovered separately for each image. The recovery of the parameters 
in the second and third stages is relatively straightforward, therefore the emphasis is on the computation of the NIAC. The methods presented are linear and do not involve large dimension problems.

\section{Algorithm 1 Calibration of a zooming camera using the NIAC \\ 0 . Estimate the homographies between each physical plane and its image, and derive the two pairs of cir- cular points associated.}

1. Compute the NIAC and derive the parameters for $K_{1}$.

2. Compute the IAC for each image and derive the focal length $f_{i}$ representing the zoom for each image.

3. Compute the rotation $R$ and the translation $t$ for each image.

\subsection{Preliminary Stage: Circular Points Computa- tion}

The NIAC has a complex equation that defines an imaginary object. Its image is defined by requiring its real and imaginary parts to be zero. The NIAC can be computed by extending the procedure used to compute the IAC. Generally, this procedure fits a conic to a set of the circular points. $[21,11,15,12]$. The images of the circular points can be obtained by computing the homography between a physical plane and the image plane $[21,11,15]$ or from the intersection of a circle with the plane at infinity [12]. Both approaches provide the same circular points. In our case, we chose to use planar homographies to compute the circular points.

We use the images of two squares with arbitrary position, orientation and scale. The only requirement is that the planes are non-coplanar. Let us denote by $H_{1}=$ $\left[\boldsymbol{h}_{1}^{1}, \boldsymbol{h}_{2}^{1}, \boldsymbol{h}_{3}^{1}\right]$ the homography between the first plane and its image, and $H_{2}=\left[\boldsymbol{h}_{1}^{2}, \boldsymbol{h}_{2}^{2}, \boldsymbol{h}_{3}^{2}\right]$ the homography between the second plane and its image. For simplicity and without loss of generality, we can assume that the planes are located in $Z=0$ (since the IAC is invariant to the position and orientation of the camera, the relative position between the physical plane and the camera does not matter). Writing the rotation matrix in the form $R=\left[\begin{array}{lll}\boldsymbol{r}_{1} & \boldsymbol{r}_{2} & \boldsymbol{r}_{3}\end{array}\right]$, each homography can be expressed in the following form

$$
H_{j} \sim K_{1} F_{f_{j}}\left[\begin{array}{lll}
\boldsymbol{r}_{1}^{j} & \boldsymbol{r}_{2}^{j} & \boldsymbol{t}^{j}
\end{array}\right]
$$

Each model plane intersects the absolute conic $\Omega_{\infty}$ in two circular points [14] denoted $\boldsymbol{I}=[1, \mathrm{i}, 0]^{\top}$ and $\boldsymbol{J}=$ $[1,-\mathrm{i}, 0]^{\top}$. The images of these points generated by the two homographies are:

$$
\begin{cases}\boldsymbol{P}_{1}=H_{1} \boldsymbol{I}=\boldsymbol{h}_{1}^{1}+\mathrm{i} \boldsymbol{h}_{2}^{1}, & \boldsymbol{P}_{2}=H_{1} \boldsymbol{J}=\boldsymbol{h}_{1}^{1}-\mathrm{i} \boldsymbol{h}_{2}^{1} \\ \boldsymbol{P}_{3}=H_{2} \boldsymbol{I}=\boldsymbol{h}_{1}^{2}+\mathrm{i} \boldsymbol{h}_{2}^{2}, & \boldsymbol{P}_{4}=H_{2} \boldsymbol{J}=\boldsymbol{h}_{1}^{2}-\mathrm{i} \boldsymbol{h}_{2}^{2}\end{cases}
$$

In the next sections, we show how the different camera parameters can be recovered from this set of circular points.

\subsection{Stage 1: Computation of $K_{1}$}

According to the previous section, the intrinsic parameters of the camera (principal point, the aspect ratio and the skew parameter) are represented by the matrix $K_{1}$. These parameters are invariant to the translation, rotation and zooming of the camera. In our method, rather than computing directly the matrix $K_{1}$, we propose to compute the NIAC given by $\omega_{n}=\left(K_{1} K_{1}^{\top}\right)^{-1}$. Once $\omega_{n}$ is known, $K_{1}$ can be recovered uniquely by Cholesky decomposition [7].

The four points in the previous equation define an infinite number of conics. To define a unique conic we would require five points. We call the family of conics defined by four points a general pencil [14]. The locus of the centres of the general pencil is a conic whose asymptotes are parallel to the axes of the two parabolas of the pencil. This locus passes through the six mid-points of the sides of the complete quadrangle defined by the four points and the three vertices of the diagonal triangle of this complete quadrangle [14]. The geometric construction is illustrated in Figure 2.

The four non-aligned points $\boldsymbol{P}_{i}(i=1 \ldots 4)$ can be joined pairwise by six distinct lines, forming a figure called a complete quadrangle. The six mid-points $\boldsymbol{M}_{i}(i=$ $1 \ldots 6)$ of the sides of the complete quadrangle belong to the locus of the centres. They can be computed by generalizing the notion of mid-point to complex points ${ }^{1}$. The vertices $\boldsymbol{X}, \boldsymbol{Y}$ and $\boldsymbol{Z}$ of the diagonal triangle can be obtained by computing the intersection of the opposite sides of the complete quadrangle (including the two diagonals). In homogeneous notation this can be expressed as $\boldsymbol{X}=\boldsymbol{P}_{1} \boldsymbol{P}_{2} \times$ $\boldsymbol{P}_{3} \boldsymbol{P}_{4}, \boldsymbol{Y}=\boldsymbol{P}_{1} \boldsymbol{P}_{3} \times \boldsymbol{P}_{2} \boldsymbol{P}_{4}$ and $\boldsymbol{Z}=\boldsymbol{P}_{1} \boldsymbol{P}_{4} \times \boldsymbol{P}_{2} \boldsymbol{P}_{3}$. Accordingly, the centre locus can be obtained by fitting a conic to the nine points $\boldsymbol{M}_{i}(i=1 \ldots 6), \boldsymbol{X}, \boldsymbol{Y}$ and $\boldsymbol{Z}$. Since some of the points are complex, the fitting should consider both the real and imaginary parts to be zero. For example,

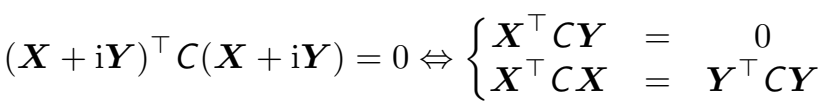

It is important to notice that the geometric construction of the locus of the centres is redundant; we have nine points and a conic can be defined by only five. A priori, any subset of size at least five can be used. In our implementation, we used the points $\boldsymbol{X}, \boldsymbol{Y}, Z, M_{1}$ and $M_{2}$. These

\footnotetext{
${ }^{1}$ If $\boldsymbol{P}_{1}$ and $\boldsymbol{P}_{2}$ are two complex points normalized such that their third coordinate is 1 , then their mid-point is the point with coordinates $\frac{1}{2}\left(\boldsymbol{P}_{1}+\right.$ $\left.\boldsymbol{P}_{2}\right)$.
} 


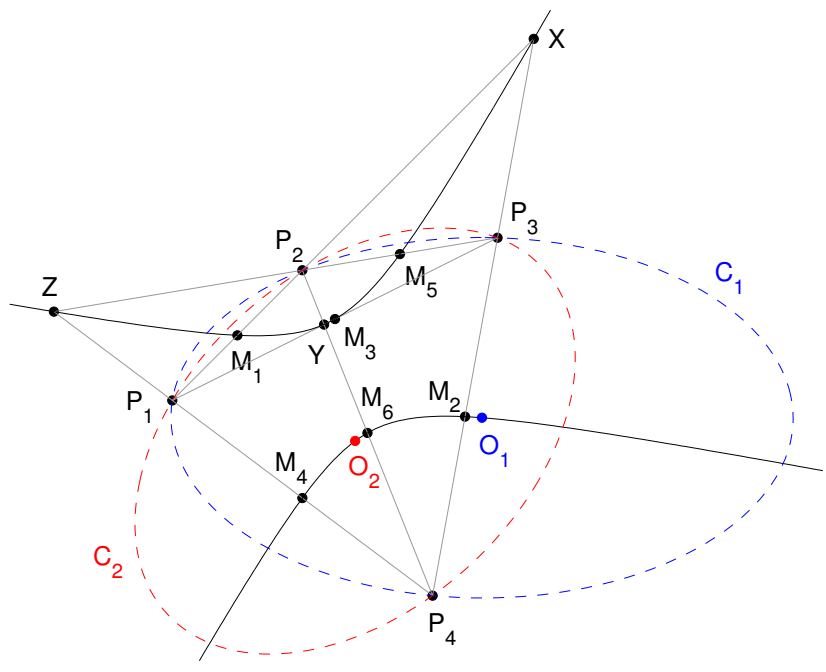

Figure 2. The locus of the centres of a general pencil of conics passing through four points. All the conics through the four points $\boldsymbol{P}_{1}, \boldsymbol{P}_{2}, \boldsymbol{P}_{3}$ and $P_{4}$ are centered on a conic which passes through the vertices $(X, Y$ and $Z$ ) of the diagonal triangle of the complete quadrangle $\boldsymbol{P}_{1} \boldsymbol{P}_{2} \boldsymbol{P}_{3} \boldsymbol{P}_{4}$ and the mid-points of the six sides of this quadrangle $\left(M_{1}, M_{2}, M_{3}, M_{4}, M_{5}\right.$ and $\left.M_{6}\right)$.

points are real (this property comes from the fact that both pairs $\left\{\boldsymbol{P}_{1}, \boldsymbol{P}_{2}\right\}$ and $\left\{\boldsymbol{P}_{3}, \boldsymbol{P}_{4}\right\}$ are conjugate), thus the fitting is generally more robust than fitting with other points and standard conic fitting techniques can be used.

If we consider the image of two calibration grids, then by fitting five points, we obtain one conic locus of the centres on which the principal point lies. If several images are available, the principal point is given by the intersection of all the conic loci (see Figure 3). Since two conics intersect in four points, we should require more than two images. However, in practice, the position of the principal point is approximately known, and this information can be used during the minimisation to compute the locus without ambiguity from two images. If more than two images are used or if more than two planes are observed, the computation of the principal point is more robust. In our implementation, the minimization is implemented by the Levenberg-Marquardt algorithm initialized at the image centre. The minimisation is performed in a space of dimension 2, thus it has a fast convergence.

Once the principal point is known, only the aspect ratio and the skew parameter need to be computed to determine the NIAC. These parameters can be obtained by fitting a conic centered in the estimated principal point to the four images of the circular points for each image. If more than two images are used, the parameters can be estimated by

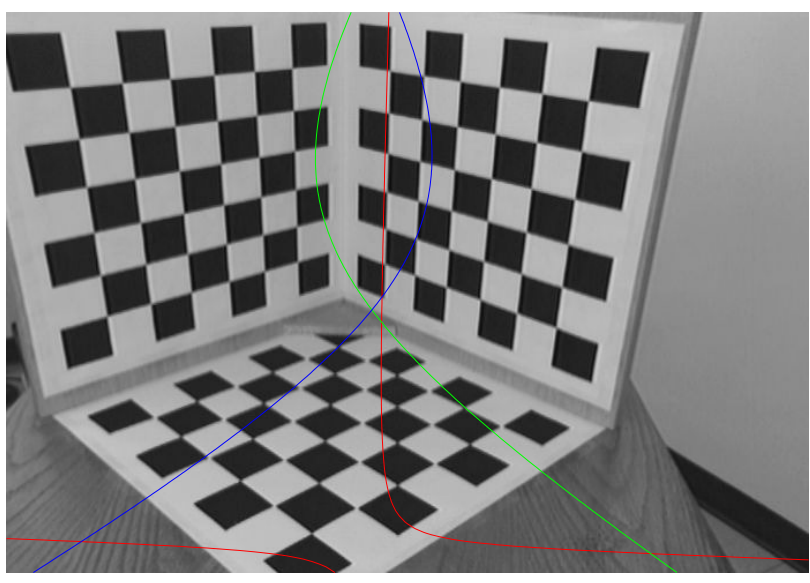

Figure 3. The conics corresponding to the locus of the centres of each pair of planes in a general position all intersect in the principal point.

combining the estimates for each pair of images. In our current implementation, the final value is obtained by averaging all the estimates.

\subsection{Stage 2: Computation of $F_{f}$}

Computing $F_{f}$ is equivalent to estimating the focal length for each image, i.e. evaluating the zoom effect. This is done by determining the scale factor of the homothety to apply to the NIAC to transform it into the IAC which best fits the four circular points for each image. A simple least square technique can be used.

\subsection{Stage 3: Computation of $R$ and $t$}

From Equation (5), we obtain a simple relation expressing the extrinsic parameters for each image:

$$
\left[\begin{array}{lll}
\boldsymbol{r}_{1}^{j} & \boldsymbol{r}_{2}^{j} & \boldsymbol{t}^{j}
\end{array}\right] \sim F_{f_{j}}^{-1} K_{1}^{-1} H_{j} .
$$

The equality is up to a scale factor. The norm of the factor can be determined by constraining the norm of the first two columns of the right hand term to be one (the columns of a rotation matrix are unit vectors). The sign is obtained by requiring the observed object to be in front of the image plane of the camera. $\boldsymbol{r}_{3}^{j}$ is obtained by the formula $\boldsymbol{r}_{3}^{j}=$ $\boldsymbol{r}_{1}^{j} \times \boldsymbol{r}_{2}^{j}$. The orthogonality of the matrix, which is usually not satisfied due to noise, can be constrained for example using the SVD decomposition and requiring each singular value to be one.

\subsection{Practical Considerations}

Normalization Ideally the results obtained should be independent of the reference frame used. However in prac- 
tice, some reference frames lead to better conditioned systems and therefore to different results. To mediate the influence of the choice of the reference frame, and to guarantee optimum results it is important to normalize the data. The normalization is done at different levels. First of all world points and images points are normalized such that their respective centroid is the origin and their average distance from the origin is $\sqrt{2}$. This was shown to give optimum results for the computation of the homographies [10]. The second normalization occurs while fitting a conic to a set of points [22] or while intersecting conics. It was shown that normalization of the data improves conic fitting in [3]. Here we use the standard technique of rescaling the columns of the equation matrix [7]. This technique was used for conic fitting in [15].

Maximum Likelihood Estimation The linear solution presented before is simple and computationally attractive. However, the distance implicitly minimized during the computation of the solution is algebraic, i. e. it is not physically meaningful. It is possible to finalise the calibration with a global non-linear minimization. The cost function being minimized is the reprojection error in each image as described in the cost function in Equation (4). The solution obtained is a maximum-likelihood estimate. In practice, the Levenberg-Marquardt algorithm is used. The rotation is parametrized by three parameters using Rodrigues formula and considering relative angles [18]. The initial guess for all the parameters is provided by the linear method described previously.

Degenerate Configurations The study of all the singularities is out of the scope of this paper. We will only mention that parallel planes do not introduce any new calibration information, since they intersect the Absolute Conic in the same Circular Points. In general, calibration is possible with two views with different orientations of the camera.

Restricted Camera Model In the case of a nonzooming camera, the method can still be applied. The second stage is replaced by computing the scale factor which transforms the NIAC into the IAC going through all the circular points from all the images simultaneously. The algorithm is more robust and can be used to decouple the parameters during calibration. In the case of a digital camera (zero skew) or if the aspect ratio is know, then the calibration can be done from a single image [21].

Case when the planar objects are known to be orthogonal It leads to a simplified algorithm, where the principal point can be computed from line intersection (not described in this paper due to space limitation).

\section{Results}

The NIAC method was tested with synthetic and real images. In both cases, the grids used were made of $8 \times 8$ con-

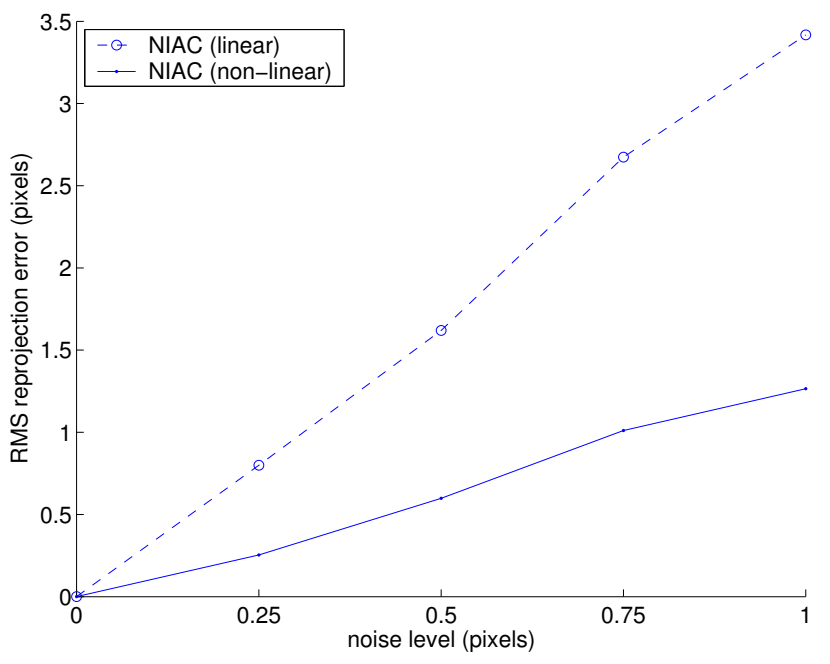

Figure 4. Results with synthetic data. Influence of the noise. The RMS reprojection error was computed from a total of 100 experiments. The noise level indicated represents the standard deviation of the zero mean Gaussian noise added in the image. 9 images were used for calibration.

trol points rather than a single square, in order to increase the robustness of the computation of the homographies. The criteria considered to evaluate the accuracy of the calibration is the Root Mean Squared (RMS) reprojection error. Two grids were used for calibration, and the RMS reprojection error was computed from a third grid added to the scene; the computation from points different from the ones used for calibration leads to more meaningful results. When a comparison is performed, the method used for comparison is the one described in [10] under the name of Gold Standard algorithm. The linear standard solution is then obtained by applying the standard method individually to each image of the subset considered and averaging the values obtained for each parameter. The results obtained in the case of the standard of the NIAC method can be refined by bundle-adjustment using a non-linear minimization algorithm (for example Levenberg-Marquardt).

\subsection{Synthetic data}

We tested the method in a general case when the two grids used for calibration are in a random unknown position. In this case the angle between the two grids was set to $100^{\circ}$.

Influence of the noise A Gaussian noise with zero mean and standard deviation $\sigma$ was added to the projected image points. The experiments were carried out with 9 images with a noise level varying from $\sigma=0$ to $\sigma=1$ pixel, and each experiment was repeated 100 times for each noise level. The results obtained are shown in Figure 4. The de- 


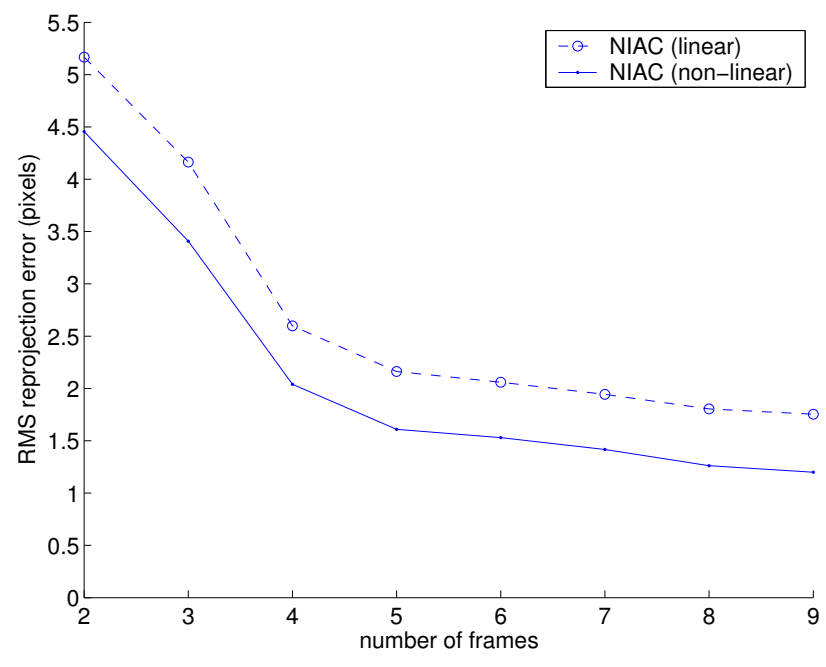

Figure 5. Results with synthetic data. Influence of the number of frames. The RMS reprojection error was computed from a total of 100 experiments. The noise in the image was Gaussian with zero mean and standard deviation $\sigma=0.5$ pixels

pendency with respect to the noise level seems to be linear.

Influence of the number of frames The influence of the number of frames used was tested by applying the method to random subsets of size varying from 2 to $9 ; 100$ experiments were carried out for each number of frames considered. The noise in the image was constant (zero mean, standard deviation $\sigma=0.5$ pixel), the results are shown in Figure 6 . The results obtained for both methods seem to converge and stabilise after 6 images.

The NIAC method seems to be accurate (reprojection errors of the order of one pixel). It can be seen that the nonlinear minimisation improves the calibration results. Here, the angle between the two planes being assumed unknown, traditional calibration methods are not applicable, so we chose not to show any comparison of the NIAC method with other standard methods. However, some additional experiments using the exact position of each control points showed that the linear standard method performed far worse than the linear NIAC method, whereas the non-linear standard method is slightly better than the non-linear NIAC. Such calibration information is usually not available, unless a high accuracy calibration grid (usually with two orthogonal planes) is used.

\subsection{Real data}

The method was tested with real images in the particular case where the calibration planes are orthogonal. This information was not used by our method, however it allows a comparison with standard calibration methods using point
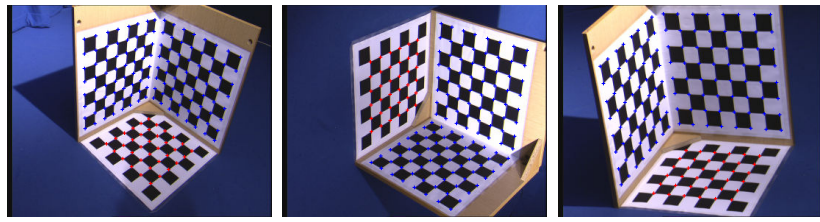

Figure 6. Three images from the sequence of 15 images with different zoom factors.

Table 2. Results for the different focal lengths estimated (the calibration was done from 9 images).

\begin{tabular}{ccccc}
\hline \hline & $\begin{array}{c}\text { linear } \\
\text { standard }\end{array}$ & $\begin{array}{c}\text { non-linear } \\
\text { standard }\end{array}$ & $\begin{array}{c}\text { linear } \\
\text { NIAC }\end{array}$ & $\begin{array}{c}\text { non-linear } \\
\text { NIAC }\end{array}$ \\
\hline$u_{0}$ & 362.6 & 362.9 & 366.3 & 366.4 \\
\hline$v_{0}$ & 307.9 & 308.0 & 280.5 & 280.4 \\
\hline$r$ & 0.9168 & 0.9162 & 0.9041 & 0.9043 \\
\hline$\theta$ & 1.570 & 1.557 & 1.572 & 1.554 \\
\hline$f_{1}$ & 801.6 & 800.9 & 794.9 & 782.0 \\
\hline$f_{2}$ & 815.7 & 814.4 & 809.9 & 813.2 \\
\hline$f_{3}$ & 806.0 & 802.9 & 795.5 & 799.1 \\
\hline$f_{4}$ & 1035.3 & 1034.5 & 1006.8 & 1022.2 \\
\hline$f_{5}$ & 1015.1 & 1015.2 & 1026.2 & 1013.4 \\
\hline$f_{6}$ & 1017.8 & 1016.6 & 998.1 & 999.8 \\
\hline$f_{7}$ & 1257.1 & 1256.3 & 1223.9 & 1217.7 \\
\hline$f_{8}$ & 1272.9 & 1269.8 & 1258.7 & 1263.8 \\
\hline$f_{9}$ & 1256.2 & 1239.2 & 1225.1 & 1227.4 \\
\hline $\mathrm{RMS}$ & 28.459 & 5.727 & 4.196 & 4.356 \\
\hline \hline
\end{tabular}

correspondences. The images were obtained from a handheld camera, at different positions, orientations and with different zoom factors. The zoom factor was fixed for consecutive triplets of images. A sequence of 15 images was used (see Figure 6).

Table 2 shows the values obtained for the intrinsic parameters and the RMS reprojection error in the case of calibration from 9 images. The values obtained for each method are very close. We can verify that triplets of images present similar values for the focal length. The results obtained with respect to the number of frames considered are shown in Figure 7. It can be seen that the NIAC method and the standard method lead to similar results.

\section{Conclusions and Further Work}

We have presented a technique for calibrating a zooming camera that considers the invariance properties of the NIAC to simplify the calibration equations. We have shown that the geometrical properties of the NIAC can be used to calibrate a camera from two images of non-coplanar squares. The method decomposes the calibration problem in three 


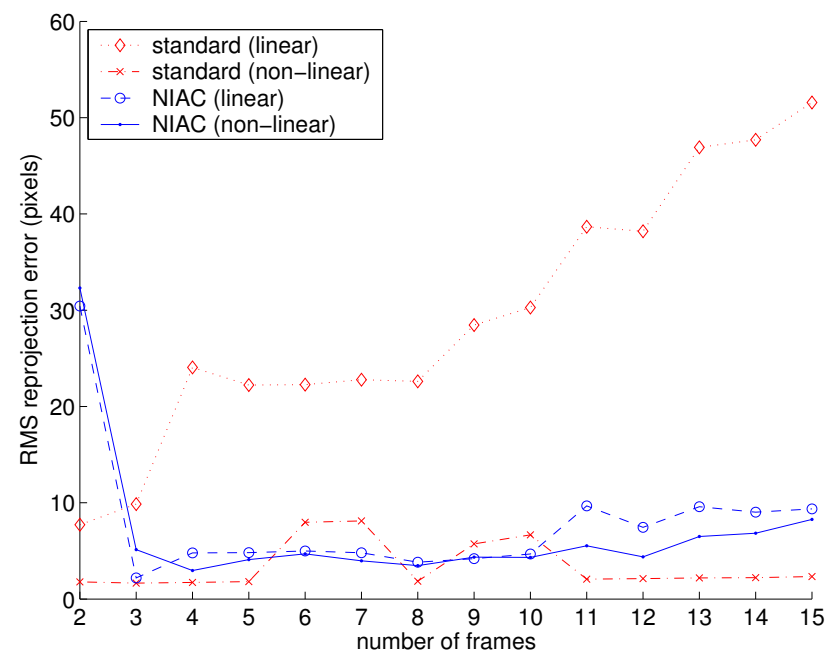

Figure 7. Results with real data. Influence of the number of frames.

sub-problems of low dimension that are solved linearly. The solution can be refined by including a non-linear minimisation step. Preliminary results with synthetic and real images show that the technique is accurate. The main limitation is that it assumes a fixed position of the principal point. This is only valid for high quality cameras and relative small zooming. More experiments that evaluate the performance as a function of the zooming factor should be done. Our current work focuses on exploring new invariance properties compatible with variable principal points: for example, the NIAC should no longer be centered at the principal point, but would be an imaginary object floating in the image space.

\section{Acknowledgements}

The authors would like to thank Joel Mitchelson for his help during image acquisition. This work is supported by EPSRC project grant number GR/R08629/01.

\section{References}

[1] R. Atienza and A. Zelinski. A practical zoom camera calibration technique: An application of active vision for human.

[2] J. Batista, P. Peixoto, and H. Araujo. Real-time active visual surveillance by integrating peripheral motion detection with foveated tracking. In In Workshop on Visual Surveillance (in conjunction with ICCV98), 1998.

[3] F.L. Bookstein. Fitting conic sections to scattered data. Computer Graphics and Image Processing, 9:56-71, 1979.
[4] B. Caprile and V. Torre. Using vanishing points for camera calibration. IJCV , 4:127-140, 1990.

[5] R. Cipolla, D.P. Robertson, and E.G. Boyer. Photobuilder $3 \mathrm{~d}$ models of architectural scenes from uncalibrated images. In IEEE International Conference on Multimedia Computing and Systems, volume 1, pages 25-31, 1999.

[6] L. de Agapito, E. Hayman, and I.D. Reid. Self-calibration of a rotating camera with varying intrinsic parameters. In BMVC, pages 883-893, 1998.

[7] G.H. Golub and C.F. Van Loan. Matrix Computations. The Johns Hopkins University Press, 1983.

[8] J.-Y. Guillemaut, A.S. Aguado, and J. Illingworth. Using points at infinity for parameter decoupling in camera calibration. In BMVC, volume 1, pages 263-272, 2002.

[9] R.I. Hartley, L. de Agapito, I.D. Reid, and E. Hayman. Camera calibration and the search for infinity. In $I C C V$, pages 510-517, 1999.

[10] R.I. Hartley and A. Zisserman. Multiple View Geometry in Computer Vision. Cambridge University Press, 2000.

[11] D. Liebowitz, A. Criminisi, and A. Zisserman. Creating architectural models from images. In Proc. EuroGraphics, volume 18, pages 39-50, September 1999.

[12] X. Meng and Z. Hu. A new easy camera calibration technique based on circular points. Pattern Recognition, 36:1155-1164, 2003.

[13] M. Pollefeys, R. Koch, and L.J. Van Gool. Self-calibration and metric reconstruction in spite of varying and unknown internal camera parameters. In ICCV, pages 90-95, 1998.

[14] J.G. Semple and G.T. Kneebone. Algebraic Projective Geometry. Oxford University Press, first edition, 1952.

[15] P. F. Sturm and S. J. Maybank. On plane-based camera calibration: A general algorithm, singularities, applications. In CVPR, pages 432-437, 1999.

[16] M. Trajkovic. Interactive calibration of a pan-tilt-zoom (ptz) camera for surveillance applications. In Asian Conference Computer Vision, 2002.

[17] B. Triggs. Autocalibration and the absolute quadric. In CVPR, pages 609-614, 1997.

[18] B. Triggs, P. McLauchlan, R. Hartley, and A. Fitzgibbon. Bundle adjustment - A modern synthesis. In W. Triggs, A. Zisserman, and R. Szeliski, editors, Vision Algorithms: Theory and Practice, LNCS, pages 298-375. Springer Verlag, 2000.

[19] R.G. Willson. Modeling and calibration of automated zoom lenses. In Proceedings of the SPIE \#2350:Videometrics III, pages 170-186, 1994.

[20] R.G. Willson and S.A. Shafer. What is the center of the image? J. Opt. Soc. Am. A, 11(11):2946-2955, 1994.

[21] Z. Zhang. A flexible new technique for camera calibration. IEEE Trans. PAMI, 22(11):1330-1334, 2000.

[22] Zhengyou Zhang. Parameter estimation techniques: A tutorial with application to conic fitting. Technical Report RR2676, INRIA, 1995. 